\title{
Development Analysis and Competitiveness Evaluation of Cultural Industry in Northwest China
}

\author{
Yuxia $\mathrm{Hu}^{1, \mathrm{a}}$ and Haiying $\mathrm{Ma}^{2, \mathrm{~b}^{*}}$ \\ ${ }^{1,2}$ School of Economics, Northwest Minzu University \& The Provincial Key Laboratory of \\ E-commerce of Ethnic Information, Northwest Minzu University, Lanzhou (730124), P.R.China
}

a2253801927@qq.com, blxmahaiying8888@163.com

Keywords: Northwest; Cultural Industry; Principal Component Analysis; Evaluation

\begin{abstract}
This paper uses the principal component analysis method to compare the comprehensive competitiveness of the cultural industries in the northwestern provinces and regions across the country, the results show that the overall ranking of regional cultural industry development is behind, and there are significant non-equilibrium among the provinces and regions within the region. Finally, from the aspects of location disadvantage, factor input and institutional environment of industrial development, the problems in the development of the northwest cultural industry are analyzed and corresponding policy recommendations are drawn.
\end{abstract}

\section{Introduction}

The Central Committee of the Communist Party of China formally proposed the concept of cultural industry in The 10th Five-Year Plan proposal in 2000. The report also mentions a sound modern cultural industrial system and market system, innovative production and management mechanisms, and improved cultural and economic policies to cultivate new forms of culture. It is an era that shapes Chinese civilization and cultural awareness, stimulates cultural creativity and industrial potential throughout the country. The inheritance of the cultural heritage conferred on the northwest region has important practical significance for the analysis of the status quo of the cultural industry development and the evaluation of comprehensive competitiveness.

Quantitative studies of industrial efficiency and competitiveness in China are mostly concentrated in the manufacturing, financial, and service industries, and less research is involved in cultural industries. Hou (2008), Ma and Zheng (2010), Zhang (2009), Jiang Ping and Wang Yong (2011), etc. analyzed the input-output efficiency of cultural industries in 31 provinces in China through different empirical models. All show that the efficiency of cultural industries needs to be improved and there are obvious efficiency gaps among different provinces. Zhao (2006), Wang Yu and Zhao Guojie (2009) constructed a competitiveness analysis index system based on different focuses, and empirically evaluated the competitiveness of cultural industries in various provinces in China. Guo and Zheng (2009) used data envelopment technology (DEA) to evaluate the development performance of the cultural industries in the six central provinces, and used the structural equation model to analyze the input-output path of the cultural industry. In our limited academic perspective, we have not yet found a study on the evaluation of the competitiveness of cultural industries in the northwest region.

This paper adopts the principal component analysis method to construct an evaluation index system. Comparing the comprehensive competitiveness of the cultural industry in the northwest region, the paper assesses the regional development status and competitive rankings and analyzes the difficulties in the development of the cultural industry in the northwest region, and the conclusions and corresponding policy recommendations will be drawn in the last section.

\section{Evaluation of Cultural Industry Competitiveness in Northwest China}

The competitiveness of the cultural industry stems from the development capabilities of cultural demand activities and cultural supply activities. The output of cultural industries can to a certain extent present 
the competitiveness of a regional cultural industry. Like other industries, the production process of the cultural industry requires capital investment in the input of cultural resources, human resources, and infrastructure. At the same time, the development of the cultural industry is inseparable from the expansion of the cultural consumer market and the cultivation of public appreciation.

Construction of Evaluation Index of Regional Cultural Industry. Based on the results of relevant cultural industry research and combined with the availability of regional data, this paper selects nine representative indicators from the three levels of cultural industry investment level, cultural industry output level, and cultural industry policy environment. The comprehensive evaluation indicators used to build the competitiveness of regional cultural industries are shown in Table 1.

Table 1 Cultural Industry Evaluation Index System

\begin{tabular}{|c|c|c|c|c|}
\hline $\begin{array}{l}\text { Target } \\
\text { layer } 1\end{array}$ & $\begin{array}{c}\text { Target layer } \\
2\end{array}$ & Target layer 3 & letter & unit \\
\hline \multirow{9}{*}{ 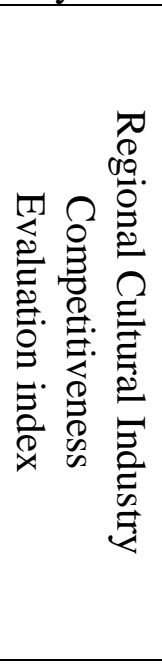 } & \multirow{4}{*}{$\begin{array}{l}\text { Cultural } \\
\text { industry } \\
\text { investment } \\
\text { level }\end{array}$} & Cultural business completed basic investment & I & $\begin{array}{l}10000 \\
\text { Yuan }\end{array}$ \\
\hline & & Number of employees & M & person \\
\hline & & Number of cultural institutions & $\mathrm{N}$ & per \\
\hline & & Number of educational institutions & A & per \\
\hline & \multirow{3}{*}{$\begin{array}{c}\text { Cultural } \\
\text { industry } \\
\text { output level }\end{array}$} & $\begin{array}{l}\text { Total income of affiliated institutions of } \\
\text { cultural relics departments }\end{array}$ & Y & $\begin{array}{l}10000 \\
\text { person }\end{array}$ \\
\hline & & Public library total inventory & B & 10000 unit \\
\hline & & Performances & $\mathrm{P}$ & 1000 unit \\
\hline & \multirow{2}{*}{$\begin{array}{c}\text { Cultural } \\
\text { Industry } \\
\text { Policy } \\
\text { Environment }\end{array}$} & Cultural per capita fee & $\mathrm{C}$ & Yaun \\
\hline & & $\begin{array}{l}\text { Cultural undertakings accounting for the } \\
\text { proportion of fiscal expenditure }\end{array}$ & $\mathrm{CP}$ & $\%$ \\
\hline
\end{tabular}

Principal Component Analysis. There are many factors in the evaluation of the development of the cultural industry, and there may be a large correlation between different indicators, which in turn makes the data information overlapping, which is not conducive to the interpretation of the true characteristics of industrial development. In order to effectively enrich the data information, we use the principal component analysis method to reduce the dimension, evaluate the development status of the cultural industry with unrelated low-dimensional information, and simultaneously obtain the weights of the evaluation indicators.

Principal component analysis (PCA) was proposed by Hotelling. This method uses projection to reduce the data dimension based on maximum use of data information, and constructs a comprehensive index that uses a small number of indicators to represent numerous data information.

Assume that there are a total number of indicators $\left(X_{1}, X_{2}, \ldots, X_{p}\right)$ that can be used to measure a problem, and thus can constitute a random vector $X=\left(X_{1}, X_{2}, \ldots, X_{p}\right)^{\prime}$, whose mean vector is $\mu$ and the covariance matrix is $\Sigma$. The vector is transformed linearly to get a synthesized random vector $Y=\left(Y_{1}, Y_{2}, \ldots, Y_{p}\right)^{\prime}$

where:

$$
\left[\begin{array}{l}
Y_{1} \\
Y_{2} \\
\ldots \\
Y_{p}
\end{array}\right]=\left[\begin{array}{l}
a_{11} a_{12} \ldots a_{1 p} \\
a_{21} a_{22} \ldots a_{2 p} \\
\ldots \ldots \ldots \ldots \ldots . . \\
a_{p 1} a_{p 2} \ldots a_{p p}
\end{array}\right]\left[\begin{array}{l}
X_{1} \\
X_{2} \\
\ldots \\
X_{p}
\end{array}\right]
$$

and $\alpha_{i}=\left(\alpha_{i 1}, \alpha_{i 2}, \ldots, \alpha_{i p}\right)^{\prime}, A=\left(\alpha_{i}, \alpha_{i}, \ldots, \alpha_{p}\right)^{\prime}$, then we have

$Y=A X, i=1,2, \ldots, p$

where $\operatorname{var}\left(Y_{i}\right)=\alpha_{i}^{\prime} \Sigma \alpha_{i}, i=1,2, \ldots, p, \operatorname{cov}\left(Y_{i}, Y_{j}\right)=\alpha_{i}^{\prime} \Sigma \alpha_{j}, i=1,2, \ldots, p$ 
To make it possible to reflect more information contained in the original variables and to prevent overlapping of different information, apply the following constraints to equation (1):

$$
\alpha_{i}^{\prime} \alpha_{i}=1, i=1,2, \ldots, p \text {, that is } \alpha_{i 1}^{2}+\alpha_{i 2}^{2}+\ldots+\alpha_{i p}^{2}=1, i=1,2, \ldots, p \text {. }
$$

When the constraint is satisfied, $Y_{1}$ the variance is the largest, in the case of irrelevant, the variance is the largest.

The composition vector $Y_{1}, Y_{2}, \ldots, Y_{p}$ that meets the constraint condition is the first principal component of the original vector, the second principal component. The first principal component and the proportion of each principal component in the total variance decreases progressively. In actual research, there are still no definitive conclusions on the exact retention of several principal components. Usually, the cumulative contribution of components or the eigenvalue method is used to determine the exact principal component.

This paper uses quantitative analysis of relevant indicators from 31 provinces and regions in China's mainland in 2013. The main sources of data are the Statistical Communique on the National Economic and Social Development of the Provinces and Regions in 2013 and the Statistical Yearbook on Cultural Relics of China in 2014. In order to eliminate interference that may occur due to dimensional differences between different indicator data, the original data is standardized.

$$
\left(\frac{x_{i j}-\bar{x}_{i}}{\sqrt{\operatorname{var}\left(x_{j}\right)}}, i=1,2, \ldots, n, j=1,2, \ldots, p\right) 。
$$

First, the data is subjected to KMO test, Bartlett test, and commonness test. The test results are shown in Tables 2 and 3:

Table 2 KMO test and Bartlett test result

\begin{tabular}{|l|c|c|}
\hline \multicolumn{2}{|c|}{ Kaiser-Meyer-Olkin Measure of Sampling Adequacy. } & 0.506 \\
\hline \multirow{2}{*}{ Bartlett's Test of Sphericity } & Approx. Chi-Square & 155.803 \\
\cline { 2 - 3 } & df & 36 \\
\cline { 2 - 3 } & Sig. & 0.000 \\
\hline
\end{tabular}

Table 3 Commonness test results

\begin{tabular}{|c|c|c|}
\hline & Initial & Extraction \\
\hline $\mathrm{I}$ & 1.000 & 0.366 \\
\hline $\mathrm{M}$ & 1.000 & 0.934 \\
\hline $\mathrm{N}$ & 1.000 & 0.952 \\
\hline $\mathrm{A}$ & 1.000 & 0.683 \\
\hline $\mathrm{Y}$ & 1.000 & 0.813 \\
\hline $\mathrm{B}$ & 1.000 & 0.687 \\
\hline $\mathrm{P}$ & 1.000 & 0.755 \\
\hline $\mathrm{C}$ & 1.000 & 0.794 \\
\hline $\mathrm{CP}$ & 1.000 & 0.795 \\
\hline
\end{tabular}

The test results show that the KMO test has higher correlation between variables; Bartlett's test rejects the null hypothesis that the correlation matrix is an identity matrix, and there is a significant correlation between variables; the commonality among variables is high, and the selected variables are adapted to the principal components.

The cumulative contribution of the total variance explained in Table 4 can be obtained. The eigenvalues of the first three principal components are greater than one, and their cumulative contribution is $75.313 \%$. Therefore, three principal components can be used to reflect the information contained in the original nine indicators. 
Table 4 Composition contribution table

\begin{tabular}{|c|c|c|c|c|c|c|}
\hline \multirow{2}{*}{ Component } & \multicolumn{3}{|c|}{ Initial Eigenvalues } & \multicolumn{3}{c|}{ Extraction Sums of Squared Loadings } \\
\cline { 2 - 7 } & Total & \% of Variance & Cumulative \% & Total & \% of Variance & Cumulative \% \\
\hline 1 & 3.657 & 40.636 & 40.636 & 3.657 & 40.636 & 40.636 \\
\hline 2 & 1.940 & 21.559 & 62.195 & 1.940 & 21.559 & 62.195 \\
\hline 3 & 1.181 & 13.118 & 75.313 & 1.181 & 13.118 & 75.313 \\
\hline 4 & .807 & 8.970 & 84.283 & & & \\
\hline 5 & .518 & 5.758 & 90.042 & & & \\
\hline 6 & .401 & 4.450 & 94.492 & & & \\
\hline 7 & .251 & 2.792 & 97.284 & & & \\
\hline 8 & .209 & 2.326 & 99.610 & & & \\
\hline 9 & .035 & .390 & 100.000 & & & \\
\hline
\end{tabular}

By dividing the number of loads in the factor load matrix by the square root of the characteristic root of the corresponding principal component, the linear combination of principal components is as follows: $Y_{1}=0.3041 I+0.4263 M+0.3550 N+0.3703 A+0.4057 Y+0.3756 B+0.3457 P-0.1388 C+0.1346 C P$ $Y_{2}=0.0719 I-0.0600 M-0.1337 N-0.2114 A+0.2278 Y+0.2962 B-0.2073 P+0.6106 C+0.6110 C P$ $Y_{1}=0.1213 I-0.4715 M-0.6215 N+0.2833 A+0.3063 Y+0.0212 B+0.4454 P-0.0121 C-0.0598 C P$

Table 5 Factor loading matrix

\begin{tabular}{|c|c|c|c|}
\hline & \multicolumn{3}{|c|}{ Component } \\
\cline { 2 - 4 } & 1 & 2 & 3 \\
\hline I & 0.582 & 0.100 & 0.132 \\
\hline M & 0.815 & -0.084 & -0.512 \\
\hline N & 0.679 & -0.186 & -0.675 \\
\hline A & 0.708 & -0.295 & 0.308 \\
\hline Y & 0.776 & 0.317 & 0.333 \\
\hline B & 0.718 & 0.413 & 0.023 \\
\hline P & 0.661 & -0.289 & 0.484 \\
\hline C & -0.265 & 0.850 & -0.013 \\
\hline CP & 0.257 & 0.851 & -0.065 \\
\hline
\end{tabular}

Based on these three principal component variance contribution rates, the sum of all indicator weights is set to 1 by normalization, and a comprehensive scoring model is obtained:

$Y=0.1184 I+0.0752 M+0.0258 N+0.1084 A+0.1941 Y+0.1675 B+0.1177 P+0.0564 C+0.1365 C P$

Using the standardized data substituting sub-item (5), the results of evaluating the competitiveness of cultural industries in 31 provinces in China are shown in Table 6.

Table 6 Comprehensive scores of cultural industry competitiveness of northwest in China

\begin{tabular}{|c|c|c|}
\hline Ranking & Regions & Scores \\
\hline 1 & Shanxi & 0.5239 \\
\hline 2 & Inner Mongolia & -0.0827 \\
\hline 3 & Xingiang & -0.2335 \\
\hline 4 & Gansu & -0.3760 \\
\hline 5 & Guizhou & -0.6037 \\
\hline 6 & Ninxia & -0.7097 \\
\hline 7 & Qinghai & -0.7260 \\
\hline 8 & Tibetan & -0.8601 \\
\hline
\end{tabular}

The overall development of cultural industries in various provinces and regions in Northwest China lags behind. As can be seen from Table 3-6, Northwest China ranks in the middle and late positions in the comprehensive score of cultural industries. Shaanxi Province has a better development of cultural industries, ranks 13th in the country, and reflects the competitiveness of cultural industries above average. The value of comprehensive competitiveness is positive. The competitiveness of cultural 
industries in Gansu, Xinjiang, Ningxia, and Qinghai are below average, and the comprehensive competitiveness is less than zero.

\section{Conclusions and Policy Implications}

To accelerate the development of the cultural industry in the new situation, the northwestern region must combine its own advantages in resources, guide the needs of the cultural industry, shape the core competitiveness of the regional cultural industry, and construct the unique culture of the northwest region, making full use of tourism, exhibitions, performing arts, media, etc. A variety of platform media, broaden the breadth of cultural resources in the northwest and cultivate the depth of cultural features. Local governments should further increase their investment in the cultural industry infrastructure, build and improve the regional public service network system, make full use of regional education resources, strengthen cooperation between the cultural industry and universities, strengthen personnel training, and build a cultural industry talent database. In addition, it is still necessary to strengthen inter-provincial communication and exchanges, break ethnic and religious segregation, and take the One Belt and One Road strategy as an opportunity to implement the establishment of the Strategic Alliance for Cultural Development of Five Provinces and Five Provinces in Northwest China set up in 2014. The region strives for the nation's supportive policy for the cultural industry in the northwest, integrates the overall image of the cultural industry in the "Silk Road" in the northwest, builds a coalition of cultural industries in the northwest, promotes the exchange of regional culture, and forms a joint force for cultural industry development.

\section{Acknowledgements}

This work is supported by The Funds of Central University of Northwest Minzu University (Grant No: 31920170059) and by the Provincial Key Laboratory of E-commerce of Ethnic Information of Northwest Minzu University and Comprehensive experimental teaching center in Economics (Grant No. 2016XJSYJXSF2X-01-10019109).

\section{References}

[1] Liu Jifa, Cultural Industry, Economic Management Press, (2005).

[2] Hu Huilin, Cultural Industry, Higher Education Press, (2006).

[3] Hua Jian, Connotation, Structure and Strategic Emphases of Cultural Industry Competitiveness, Journal of Peking University: Philosophy and Social Sciences, 2 (2005) 9-16.

[4] Li Yichun, A Preliminary Study on Evaluation Index System of Provincial Cultural Industry Competitiveness--A Case Study of Anhui Province, Comparative Economic and Social Systems, 2 (2006) 99-103.

[5] Hou Yanhong, Research on Evaluation of Cultural Industry Investment Performance, Tianjin Polytechnic University,( 2008).

[6] Ma Yi, Zheng Shilin, A review and prospect of the research on regional cultural industry efficiency in China, Economics Dynamics, 3(2010)83-86.

[7] Wang Family, Zhang Rong, Research on the efficiency of cultural industries in 31 provinces and cities in China based on the three-phase DEA model, China Soft Science, 9 (2009) 75-82.

[8] Wang Yi, Zhao Guojie, The construction of evaluation model of cultural industry competitiveness in China, Journal of Tianjin University: Social Sciences, 11(2009)14-17. 